\title{
Rol de la fuerza de trabajo renal anti-COVID-19 (FUTAC Renal Chile) en pacientes renales crónicos durante año 2020
}

\author{
Rubén Torres-Díaz ${ }^{*}$, Luis Toro ${ }^{1}$, Ma. Eugenia Sanhueza1, Eduardo Lorca², Mireya Ortiz ${ }^{3}$, \\ Jacqueline Pefaur ${ }^{4}$, Rene Clavero ${ }^{5}$, Alejandro Pacheco1,6, Eduardo Machuca7, Fernando González², \\ Patricia Herrera ${ }^{2}$, Alfredo Mocarquer ${ }^{8}$, Alondra Frías ${ }^{1}$, Eric Roessler ${ }^{3}$, Carolina Muñoz ${ }^{9}$, César Aravena ${ }^{10}$, \\ Enrique Quintana ${ }^{11}$, Juan Lemus ${ }^{12}$, Mario Lillo ${ }^{13}$, Enrique Reynolds ${ }^{2,14}$, Álvaro Morales ${ }^{2}$, Edgard País ${ }^{15}$, \\ Andrea Fabianne ${ }^{4}$, Rodrigo Mancilla ${ }^{16}$, Germana Sotomayor ${ }^{2}$, Marcela González $^{17}$, Cecilia Miranda ${ }^{18}$, \\ Eduardo Briones ${ }^{19}$, Esteban Gómez ${ }^{20}$, Sergio Mezzano ${ }^{21}$, Waldo Bernales ${ }^{22}$, Ximena Rocca ${ }^{2}$, \\ Óscar Espinoza², Eric Zuñiga ${ }^{23}$, Henry Aragón ${ }^{24}$, María Badilla ${ }^{25}$, Marcela Valenzuela ${ }^{15}$, Luis Escobar ${ }^{24}$, \\ Daniela Zamora ${ }^{4}$, Iván Flores ${ }^{26}$, Beatriz Tapia ${ }^{5}$, Tamara Bórquez ${ }^{27}$ y Patricio Herrera ${ }^{28}$
}

${ }^{1}$ Sección de Nefrología, Departamento de Medicina, Hospital Clínico, Universidad de Chile, Santiago; '2Servicio de Nefrología, Hospital del Salvador, Santiago; ${ }^{3}$ Centro hemodiálisis crónica, Pontificia Universidad Católica de Chile UC-Christus, Santiago; ${ }^{4}$ Servicio de Nefrología, Hospital Barros Luco Trudeau, Santiago; ${ }^{5}$ Servicio de Nefrología, Hospital Dr. Gustavo Fricke, Valparaíso; ${ }^{6}$ Centros de Diálisis Diaverum Chile Región Metropolitana; ${ }^{7}$ Fresenius Medical Care Chile, ${ }^{8}$ Diálisis Gran Avenida, Santiago; ${ }^{9}$ Servicio de Nefrología, Hospital de Puerto Montt, Los Lagos; ${ }^{10}$ Diálisis Municipal, La Granja, Santiago; ${ }^{11}$ Servicio de Nefrología, Hospital Padre Hurtado, Santiago; ${ }^{12}$ Diálisis Antares, Santiago; ${ }^{13}$ Centro Médico y de Diálisis Ltda., Santiago; ${ }^{14}$ Centro de diálisis crónica, Clínica Alemana, Santiago; ${ }^{15}$ Servicio de Nefrología, Complejo Asistencial Sótero del Río, Santiago; ${ }^{16}$ Servicio de Nefrología, Hospital Clínico de Magallanes, Punta Arenas; ${ }^{17}$ Servicio Nefrología, Clínica Santa María, Santiago; ${ }^{18}$ CID Servicio Integral de Salud S.A., centro de hemodiálisis, Santiago; ${ }^{19}$ Servicio de Nefrología, Clínica Indisa, Santiago; ${ }^{20}$ Servicio de Nefrología, Hospital Clínico San Borja Arriaran, Santiago; ${ }^{21}$ Servicio de Nefrología, Facultad de Medicina, Universidad Austral de Valdivia; ${ }^{22}$ Servicio de Nefrología, Hospital Regional de Coyhaique; ${ }^{23}$ Servicio de Nefrología, Clínica Bupa de Antofagasta; ${ }^{24}$ Servicio de Nefrología, Hospital San José, Santiago; ${ }^{25}$ Servicio de Nefrología, Hospital de Puerto Natales; ${ }^{26}$ Sección Nefrología, Hospital de Curicó; ${ }^{27}$ Servicio de Nefrología, Hospital Clínico del Sur, Región del Bío; ${ }^{28}$ Servicio de Nefrología, Hospital de Castro, Servicio de Salud, Isla de Chiloé. Chile

\section{Resumen}

Introducción: La enfermedad por coronavirus 2019 (COVID-19) en enfermos renales crónicos (ERC) en terapia de reemplazo renal (TRR), hemodiálisis (HD), diálisis peritoneal (DP) y trasplante (Tx) renal tiene elevada mortalidad, con datos clínicos y epidemiológicos en Latinoamérica aún limitados. Métodos: Estudio nacional observacional, multicéntrico, obtenido por la fuerza de tarea contra COVID-19 en Chile (FUTAC Renal), evaluando epidemiología y resultados clínicos mayores de los pacientes ERC en TRR, que desarrollaron COVID-19 en Chile el año 2020. Resultados: De 21,021 pacientes en HD, 1,498 en DP y 4,305 Tx renales, la tasa de infección acumulada a diciembre 2020 fue del 12.8, 7.0 y 5.2\%, y la letalidad acumulada del 20.0, 21.9 y 16.0\% respectivamente. La tasa de hospitalización por COVID-19 en cualquier TRR fue del 50\%. Los predictores mayores de mortalidad en HD fueron: edad > 60 años (odds ratio [OR]: 2.69), diabetes (OR: 1.58) y vivir en Santiago (OR: 1.67). En DP: edad > 60 años, mas de cinco años en diálisis y diabetes mellitus. En Tx renal: edad > 60 años y bajo nivel socioeconómico. Conclusiones: La COVID-19 en ERC en TRR en Chile tiene un componente sindémico, junto a una elevada tasa de contagio y letalidad, datos que permitieron a FUTAC Renal sensibilizar a autoridades de salud para su vacunación prioritaria.

Palabras clave: Hemodiálisis. Diálisis peritoneal. Trasplante renal. COVID-19. SARS-CoV-2.

\section{Correspondencia:}

*Rubén Torres-Díaz

E-mail: rtorresnefro@gmail.com
Fecha de recepción: 02-10-2021

Fecha de aceptación: 23-10-2021

DOI: 10.24875/NEFRO.21000045
Disponible en internet: 27-12-2021

Nefro Latinoam. 2021;18:128-136 www.nefrologialatinoamericana.com 2444-9032/○ 2021 Sociedad Latinoamericana de Nefrología e Hipertensión. Publicado por Permanyer. Este es un artículo open access bajo la licencia CC BY-NC-ND (http://creativecommons.org/licenses/by-nc-nd/4.0/). 


\section{Role of the anti-COVID-19 renal taskforce (FUTAC Renal Chile) in chronic kidney patients during 2020}

\section{Abstract}

Introduction: COVID-19 in chronic kidney disease (CKD) in renal replacement therapy (RRT), hemodialysis (HD), peritoneal dialysis (DP), and kidney transplant (Tx), has high mortality, with clinical and epidemiological data in Latin America still limited. Methods: National observational, multicenter study, with data obtained by the Task Force against COVID-19 in Chile (FUTAC Renal), evaluating epidemiology and major clinical results of CKD patients in RRT, who developed COVID-19 in Chile in 2020. Results: Of 21,021 patients on HD, 1,498 on DP and 4,305 renal TX, the accumulated infection rate in 2020 was $12.8 \%, 7.0 \%, 5.2 \%$, and the accumulated fatality $20.0 \%, 21,9 \%$ and $16.0 \%$ respectively. The COVID-19 hospitalization rate in all RRTs was $50 \%$. The major predictors of mortality in HD were age> 60 years (OR 2.69), diabetes (OR 1.58), and living in Santiago (OR 1.67). In DP age> 60 years, more than 5 years in therapy, and diabetes mellitus. In renal Tx age> 60 years and low socioeconomic level. Conclusions: COVID-19 in CKD in RRT in Chile, was associated with a syndemic component, and a high contagion and fatality rate, data that allowed FUTAC Renal to sensitize health authorities for priority vaccination of these patients.

Key words: Hemodialysis. Peritoneal Dialysis. Kidney transplantation. COVID-19. SARS-CoV-2.

\section{Introducción}

En diciembre de 2019 en Wuhan, China, se identificó una serie de pacientes con enfermedad respiratoria aguda de origen desconocido ${ }^{1,2}$. Los distintos estudios detectaron que la enfermedad estaba causada por un nuevo betacoronavirus al que se denominó coronavirus 2 del síndrome respiratorio agudo grave (SARS-CoV-2). La infección por SARS-CoV-2 rápidamente se esparció desde Wuhan a otras áreas del mundo, en particular a Europa, con Italia y España liderando el número total de $\operatorname{casos}^{3-6}$. El 11 de febrero de 2020, la Organización Mundial de la Salud (OMS) cambió oficialmente el nombre de la enfermedad causada por el SARS-CoV-2 a enfermedad por coronavirus 2019 (COVID-19) y tras la rápida propagación mundial, el 11 marzo 2020 fue declarada por la OMS como pandemia ${ }^{7}$. En Chile, el primer caso de COVID-19 en la población general fue diagnosticado el 3 de marzo de 2020, en un paciente que había regresado del sudeste asiático. Esto fue seguido por un incremento progresivo de los casos y fallecimientos en alrededor de cuatro olas de la epidemia, que se combatieron con varias intervenciones para tratar de minimizar el efecto de la enfermedad en Chile, que incluyeron: plan paso a paso, que correspondía a aislamiento progresivo según numero de pacientes contagiados; el desarrollo de trazabilidad de casos positivos y sospechosos por medio del sistema Epivigila; y vacunación progresiva de la población. A 13 de octubre de 2021 en Chile hay 1,664,725 casos diagnosticados con COVID-19 y han fallecido 37,578 personas a consecuencia de esta enfermedad (letalidad del $2.22 \%)^{8}$.
Sabíamos que la pandemia iba a imponer una carga mundial sin precedentes en los sistemas sanitarios, con una demanda de cuidados críticos que excederían la capacidad de atención. Lo anterior, sumado al hecho que aun no existían vacunas, ni tratamientos comprobados que fueran efectivos para este tipo de infección viral, hacía vislumbrar un escenario muy complejo para nuestros pacientes en diálisis y trasplante (Tx) renal. Estos pacientes tienen una mayor probabilidad de desarrollar complicaciones graves al adquirir COVID-19 por múltiples razones, tales como edad avanzada, múltiples comorbilidades como enfermedades cardiovasculares, hipertensión arterial, diabetes, además de tener un estado de compromiso inmunitario subyacente que se asocia a un mayor riesgo de morbimortalidad cuando se contagian con COVID-199,10. Además el riesgo de contagio puede ser especialmente mayor en los pacientes en hemodiálisis (HD), no solo por sus comorbilidades (edad avanzada y diabetes), sino debido a la necesidad de asistir trisemanalmente a centros de atención médica, requiriendo de interacciones de rutina con el personal de atención médica y otros pacientes en un espacio cerrado y compartido, por lo que es fundamental implementar estrategias preventivas con el fin de disminuir precozmente los riesgos de contagio de los pacientes en este tipo de terapias ${ }^{11,12}$.

La primera publicación de enfermedad renal crónica (ERC) en HD se realizó en un solo centro de HD, en el Hospital Renmin de la Universidad de Wuhan (abril 2020), que reportó 37 casos de COVID-19 entre 230 pacientes en HD (16\%), la mayoría con síntomas leves, sin necesidad de ingreso en unidades de cuidados 
intensivos ${ }^{13}$. Sin embargo, este hallazgo no fue concordante con el encontrado en series italianas y españolas, donde fallecieron alrededor de un $28 \%$ de los pacientes en HD crónica con COVID-19 que requirieron hospitalización $^{14,15}$. Los datos publicados de pacientes hemodializados en Guatemala que desarrollaron COVID-19, demostraron un $27.7 \%$ de letalidad, lo que confirma el alto riesgo de esta enfermedad en este grupo de pacientes ${ }^{16}$.

Frente al enorme desafío que significó enfrentar esta pandemia en nuestro país, la Sociedad Chilena de Nefrología, en conjunto con la Fundación Pro Salud Renal, la Asociación de Pacientes Dializados y Trasplantados de Chile, y la Sociedad de Enfermería en Diálisis y Trasplante, creó el 29 de marzo 2020 una Fuerza de Trabajo Conjunta Renal Anti COVID-19 (FUTAC Renal), con el objetivo de desarrollar y potenciar todas aquellas medidas para evitar la propagación de la COVID-19 en pacientes con patología renal y en el personal de salud que los atiende. Los principales objetivos de la FUTAC Renal en Chile fueron:

1. Potenciar y ayudar a instalar las barreras sanitarias en todas las unidades y equipos de salud renal del país, en especial en HD.

2. Optimizar el diagnóstico precoz de infección por COVID-19 de los pacientes renales crónicos, en especial en HD.

3. Instalar los mejores modelos de atención de salud necesarios, en todas las unidades y equipos de salud renal del país, mediante la creación de guías clínicas de manejo del COVID-19 en estos pacientes.

4. Evidenciar las brechas actuales en todas las unidades y equipos de salud renal del país, que atienden a pacientes en HD y diálisis peritoneal (DP), con objeto de ayudar en subsanarlas 0 al menos en disminuirlas.

5. Dar apoyo y crear un sentido de trabajo en equipo apoyado en la evidencia médica pero a la vez empático a todas las unidades y equipos de salud renal del país, y a nuestros pacientes en diálisis y trasplante renal.

6. Realizar el seguimiento epidemiológico de los pacientes y personal que atiende a pacientes con ERC terminal en HD, DP y Tx renales, pues consideramos que desarrollar un registro prospectivo de datos era fundamental para analizar el efecto de la pandemia por COVID-19 en pacientes con ERC en terapia de reemplazo renal (TRR) en Chile y llegar a las autoridades de salud del país ${ }^{17,18}$.

A partir de este registro presentamos la epidemiología y resultados clínicos mayores (hospitalización y mortalidad) de los pacientes con ERC en TRR que desarrollaron COVID-19 en Chile durante el año 2020, haciendo además un análisis de los principales predictores de mortalidad en esta población.

\section{Métodos}

\section{Diseño del estudio}

Chile es un país donde existen tres sistemas de atención de salud: público (FONASA), privado (ISAPRES) y Fuerzas Armadas, siendo el sistema público el principal financiador de los pacientes con ERC en TRR. Según registros de la Sociedad Chilena de Nefrología y de la Sociedad Chilena de Trasplante, hasta diciembre del año 2020 se contabilizó un total de 29,157 pacientes con ERC en TRR. A partir de los datos generados por FUTAC Renal Chile, presentamos este registro nacional de los adultos con diagnóstico de COVID-19 (reacción en cadena de la polimerasa con transcriptasa inversa [RT-PCR] positiva o imágenes características de SARS-CoV-2) en un total de 21,021 pacientes en HD (90\% de esa población), 1,498 pacientes en DP ( $100 \%$ de esa población) y 4,265 Tx renales ( $98 \%$ de esa población), desde marzo a diciembre del 2020.

Se analizaron en cada una de estas cohortes las tasas de infección, mortalidad y letalidad, en el periodo de tiempo indicado, comparándolas entre ellos y con la población general que presentó COVID-19.

En la población de pacientes en HD en que se logró obtener información de variables clínicas, como hospitalización, uso de ventilación mecánica, registro de fallecidos, comorbilidad y distribución geográfica del país (2,422 pacientes, $89.7 \%$ del total pacientes en HD que tuvieron COVID-19 año 2020), se realizó un análisis de los principales determinantes de mortalidad y un análisis de la posible influencia de la zona de residencia en la región metropolitana para evaluar un posible efecto sindémico de la pandemia. Este estudio fue aprobado por el Comité de Ética del Hospital Clínico de la Universidad de Chile.

Para el análisis estadístico se utilizó la prueba exacta de Fisher para variables categóricas y la prueba de Shapiro-Wilk para evaluar la distribución de las variables continuas. Las variables se expresaron como media aritmética \pm desviación estándar para variables paramétricas, y como mediana (percentil 25-percentil 75) para variables no paramétricas. Para los análisis de variables continuas, se utilizaron la prueba $t$ de Student (variables paramétricas) y la prueba $U$ de 


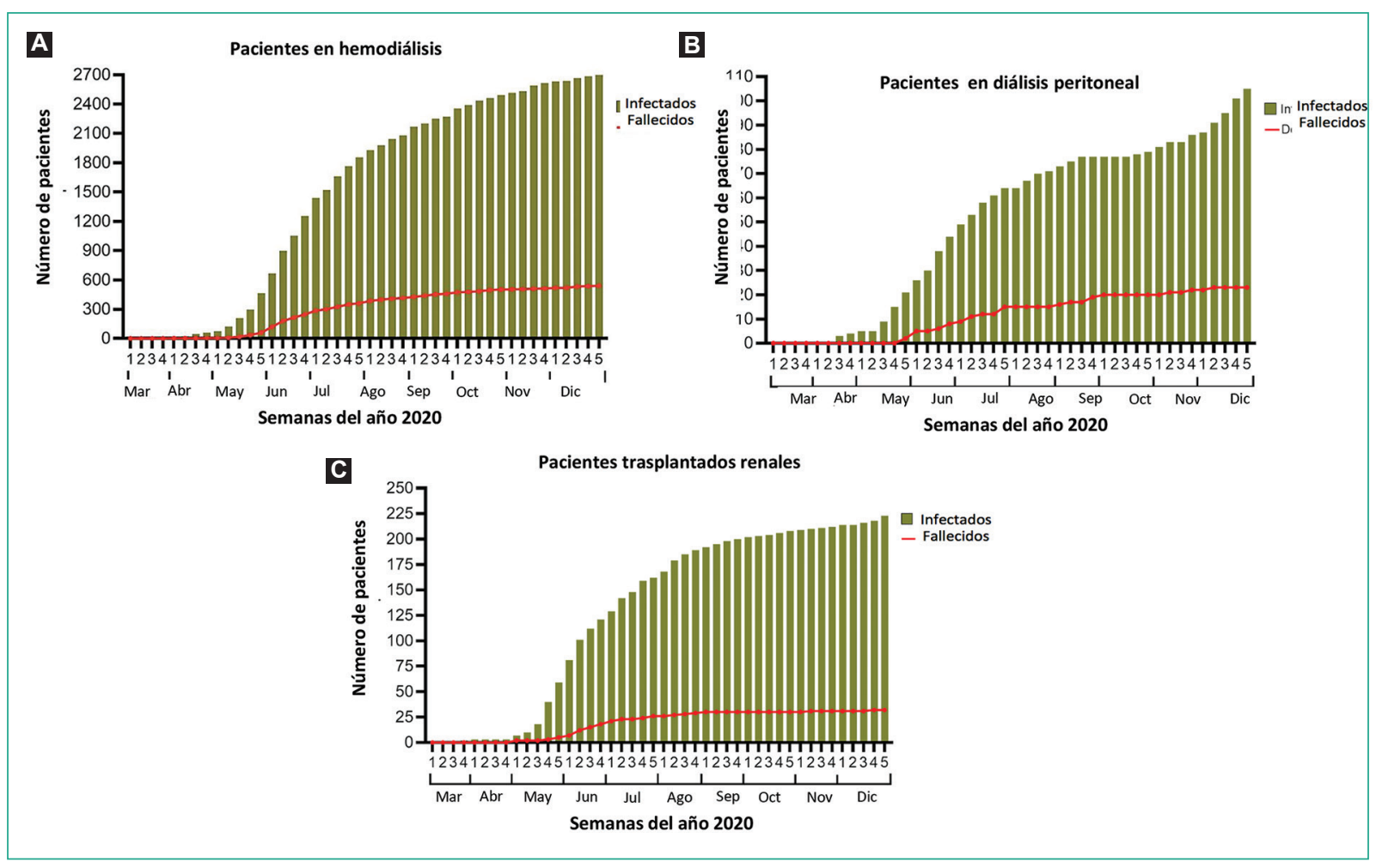

Figura 1. A: de una población total de 21,021 pacientes en hemodiálisis. B: 1,498 en diálisis peritoneal. C: 4,305 trasplantados renales con injerto funcional se grafican los casos acumulados de pacientes que desarrollaron enfermedad por coronavirus 2019 (COVID-19) y casos acumulados de pacientes que fallecieron por COVID-19 desde marzo a diciembre del 2020 en Chile.

Mann-Whitney (variables no paramétricas). El análisis multivariante se realizó mediante regresión logística multivariante, con cálculo de odds ratios (OR) e intervalo de confianza del 95\% (IC 95\%). Los datos se analizaron mediante los softwares GraphPad Prism v.6.0 (GraphPad Software, La Jolla, CA) y Stata/SE v.15.0 (Stata Software, College Station, TX). Todos los análisis fueron bilaterales y se consideró como diferencia estadísticamente significativa un grado de significación $(p)<0.05$.

\section{Resultados}

En Chile hasta diciembre de 2020 se siguió una población total de 21,021 pacientes en HD, 1,498 en DP y 4,305 Tx renales. Los pacientes que desarrollaron COVID-19 fueron 2,699 en HD (incidencia acumulada: $12.8 \%), 105$ en DP (incidencia acumulada: $7.0 \%$ ), y 225 Tx renales (incidencia acumulada: 5.2\%) (Fig. 1). De los pacientes que desarrollaron COVID-19, fallecieron 541 en HD (20.0\% de letalidad), 23 en DP (21.9\% de letalidad) y $36 \mathrm{Tx}$ renales (16.0\% de letalidad) (Fig. 2).
Al comparar las tasas de infección, mortalidad y letalidad en las diferentes cohortes de pacientes con la población general de Chile a diciembre del 2020, en HD la tasa de infección es 4.1 veces mayor, la mortalidad 30.2 veces mayor y la letalidad 7.3 veces mayor. En DP la tasa de infección es 2.2 veces mayor, la tasa de mortalidad 18.0 veces mayor y la letalidad 8.0 veces mayor. En Tx renales la tasa de infección es 1.7 mayor, la tasa de mortalidad 9.9 veces mayor y la letalidad 5.9 veces mayor (Tabla 1).

Por lo tanto, la población con mayor riesgo de desarrollar COVID-19 son los pacientes en HD, seguida de DP y al final Tx renales. Sin embargo, la letalidad es muy alta en todas las cohortes, (en HD 7.3 veces mayor, en DP 8.0 veces mayor y en Tx renales 5.9 veces mayor que la población general), demostrando que la población completa de ERC en TRR es especialmente susceptible de adquirir una forma grave de COVID-19 con elevado requerimiento de hospitalización y elevada letalidad.

Al evaluar el porcentaje de hospitalizaciones en los pacientes que desarrollaron COVID-19, este fue un $49.8 \%$ en HD, un $50 \%$ en DP y un $50 \%$ en Tx renales, vs. $9.4 \%$ en la población general del país (Fig. 2). 


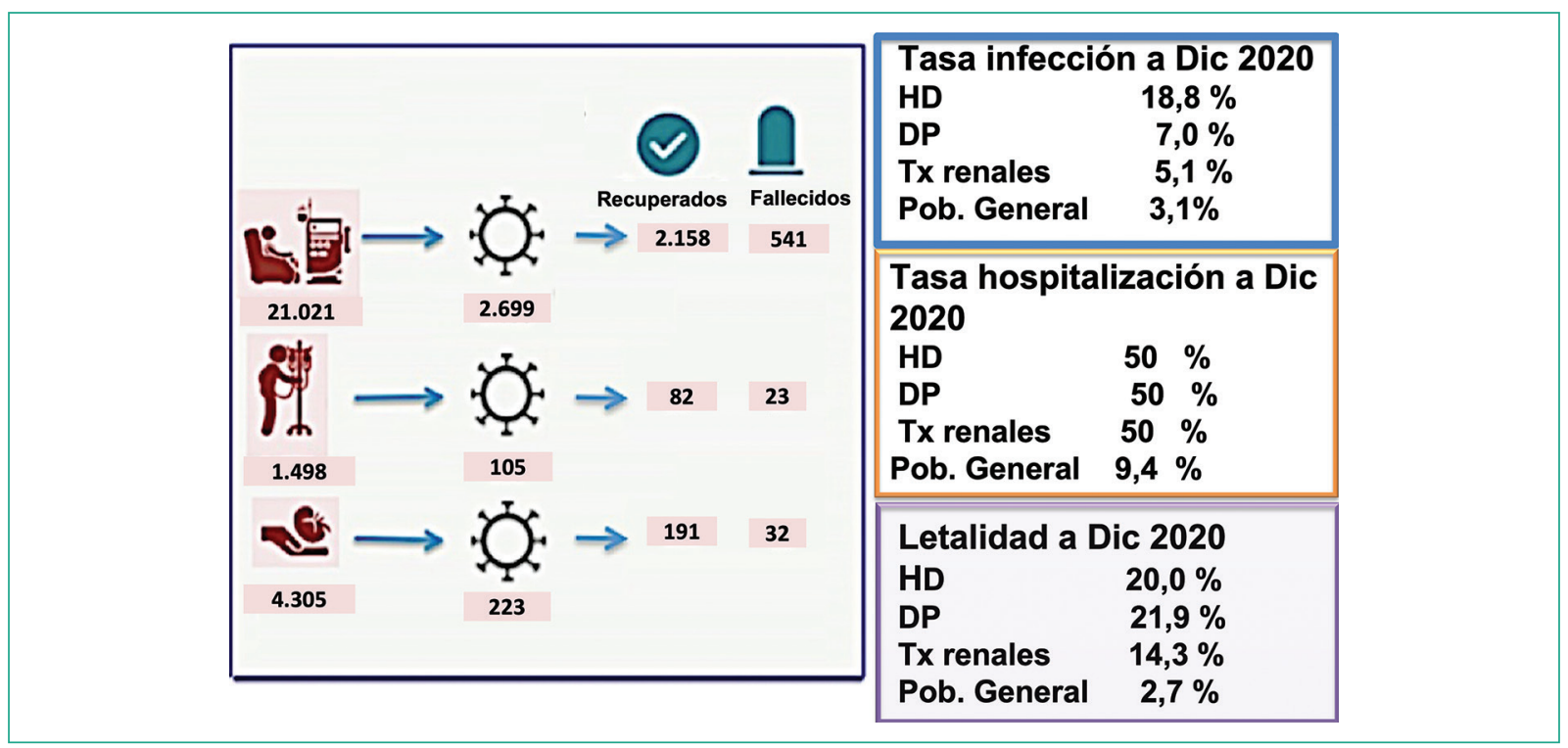

Figura 2. Resumen gráfico de tasa de infección, hospitalización y letalidad en pacientes en hemodiálisis, diálisis peritoneal y trasplantados renales, durante pandemia por enfermedad por coronavirus 2019 (COVID-19) en Chile desde marzo a diciembre del año 2020.

HD: hemodiálisis; DP: diálisis peritoneal; Tx: trasplante.

Tabla 1.Tasas de infección, mortalidad y letalidad en pacientes con enfermedad renal crónica en hemodiálisis, peritoneodiálisis y trasplante renal en Chile a 31/12/2020. Datos de pacientes en hemodiálisis, trasplante renal y peritoneodiálisis obtenidos de FUTAC (registro a 31/12/2020). Datos de población general obtenidos de MINSAL (registro del 31/12/2020)

\begin{tabular}{|c|c|c|c|c|}
\hline & $\begin{array}{l}\text { Población } \\
\text { general }\end{array}$ & $\begin{array}{l}\text { Pacientes en } \\
\text { hemodiálisis }\end{array}$ & $\begin{array}{l}\text { Pacientes con trasplante } \\
\text { renal }\end{array}$ & $\begin{array}{l}\text { Pacientes en } \\
\text { peritoneodiálisis }\end{array}$ \\
\hline Total de personas & $19,458,310$ & 21,021 & 4,305 & 1,498 \\
\hline Total de casos infectados por COVID-19 & 608,973 & 2,699 & 223 & 105 \\
\hline Total de fallecidos por COVID-19 & 16,608 & 541 & 32 & 23 \\
\hline $\begin{array}{l}\text { Tasa de incidencia de infección (por 100,000 } \\
\text { personas)* }\end{array}$ & $3,129.63$ & $12,839.54$ & $5,180.02$ & $7,009.35$ \\
\hline Tasa de mortalidad (por 100,000 personas) $^{\dagger}$ & 85.35 & $2,573.62$ & 743.32 & $1,535.38$ \\
\hline Tasa de letalidad (por 100 personas) $)^{\ddagger}$ & $2.73 \%$ & $20.04 \%$ & $14.35 \%$ & $21.90 \%$ \\
\hline
\end{tabular}

De los 2,422 pacientes con ERC en HD que desarrollaron COVID-19, en quienes se contaba con datos clínicos completos, se observa que el $55.9 \%$ eran mujeres y el $43.9 \%$ hombres, la edad promedio de los pacientes fue de $62 \pm 14.1$ años, el tiempo promedio en la terapia fue 42 meses (intervalo 17-84 meses), el $60.8 \%$ de los pacientes eran mayores de 60 años, el $48.7 \%$ tenían diabetes mellitus, el $79.5 \%$ eran hipertensos y un $4.2 \%$ usaba algún tipo de inmunosupresión. El $91.4 \%$ eran pacientes que se dializaban en centros privados de diálisis y un $8.5 \%$ en centros públicos. El $62 \%$ de los pacientes se dializaban en la región metropolitana (Santiago) y un $38 \%$ en otras regiones del país. Requirieron hospitalización 1,027 pacientes (49.8\%). De los pacientes que se hospitalizaron, un $14.9 \%$ requirió ventilación mecánica. Fallecieron directamente por COVID-19 un total de 586 pacientes (24.1\% de letalidad) (Tabla 2).

Al realizar un análisis de la distribución porcentual de los 2,422 pacientes en HD que desarrollaron 
Tabla 2. Análisis de 2,422 pacientes en hemodiálisis con datos clínicos completos, que desarrollaron enfermedad por coronavirus 2019 (COVID-19) durante 2020. Se observa que hay un $55.9 \%$ de mujeres y un $43.9 \%$ de hombres. La edad promedio de los pacientes es de 62 años, el tiempo promedio en la terapia es 42 meses. El $62 \%$ se dializan en la región metropolitana (Santiago de Chile), 1,027 pacientes se hospitalizaron $(49.8 \%)$ y 586 pacientes fallecieron por COVID-19 (24.1\% de letalidad)

\begin{tabular}{|l|c|c|}
\hline Variable analizada & \multicolumn{2}{|c|}{ Resultados } \\
\hline $\begin{array}{l}\text { Total pacientes en HD con COVID-19 } \\
\text { analizados }\end{array}$ & \multicolumn{2}{|c|}{2,422} \\
\hline Sexo masculino (n, \%) & 1,063 & $43.91 \%$ \\
\hline Edad (años, promedio \pm DE) & $61.7 \pm 14.1$ \\
\hline Edad mayor a 60 años (\%) & 1,472 & $60.80 \%$ \\
\hline Edad mayor a 65 años (\%) & 1,113 & $45.97 \%$ \\
\hline $\begin{array}{l}\text { Tiempo en hemodiálisis (meses, } \\
\text { intervalo) }\end{array}$ & 42 & $(17-84)$ \\
\hline Diabetes (n, \%) & 1,169 & $48.27 \%$ \\
\hline Hipertensión (n, \%) & 1,925 & $79.58 \%$ \\
\hline Uso de inmunosupresores (n, \%) & 102 & $4.21 \%$ \\
\hline Centro público (n, \%) & 207 & $8.55 \%$ \\
\hline Región metropolitana (n, \%) & 1,505 & $62.14 \%$ \\
\hline Hospitalizaciones (n, \%) & 1,207 & $49.83 \%$ \\
\hline $\begin{array}{l}\text { Uso ventilación mecánica en } \\
\text { hospitalizados (n, \%) }\end{array}$ & 181 & $14.99 \%$ \\
\hline Fallecidos (n, \%) & 586 & $24.19 \%$ \\
\hline
\end{tabular}

DE: desviación estándar.

COVID-19 y de los fallecidos en HD por COVID-19 según la edad en años. Se observa que en Chile existe un elevado porcentaje de pacientes sobre los 60 años (60.8\%), y que la letalidad es más alta en todos los grupos etarios, pero en especial en los mayores de 70 años, donde llegó al 35\% letalidad (Fig. 3).

Al realizar un análisis multivariante de potenciales predictores de mortalidad en pacientes en HD crónica en Chile durante la primera ola de COVID-19 (marzo a diciembre de 2020). Se observa que la edad sobre 60 años, los meses en diálisis, la presencia de diabetes mellitus y el pertenecer a la región metropolitana son todos predictores de mayor riesgo de fallecer por $\mathrm{CO}$ VID-19 (Tabla 3).

A realizar un análisis similar en la DP los principales predictores de mortalidad fueron edad sobre 60 años, tiempo en terapia mayor a cinco años y diabetes

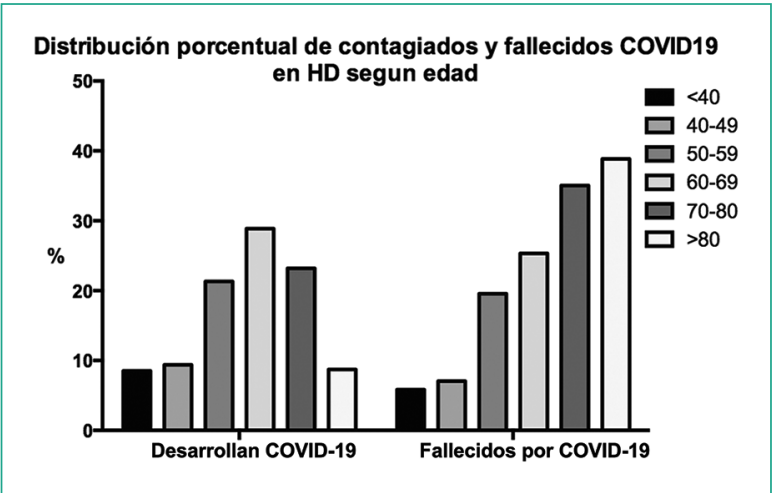

Figura 3. Distribución porcentual de 2,422 pacientes en hemodiálisis (HD) que desarrollaron enfermedad por coronavirus 2019 (COVID-19) y que fallecieron por COVID-19, según el grupo etario en que se encontraban (edad en años).

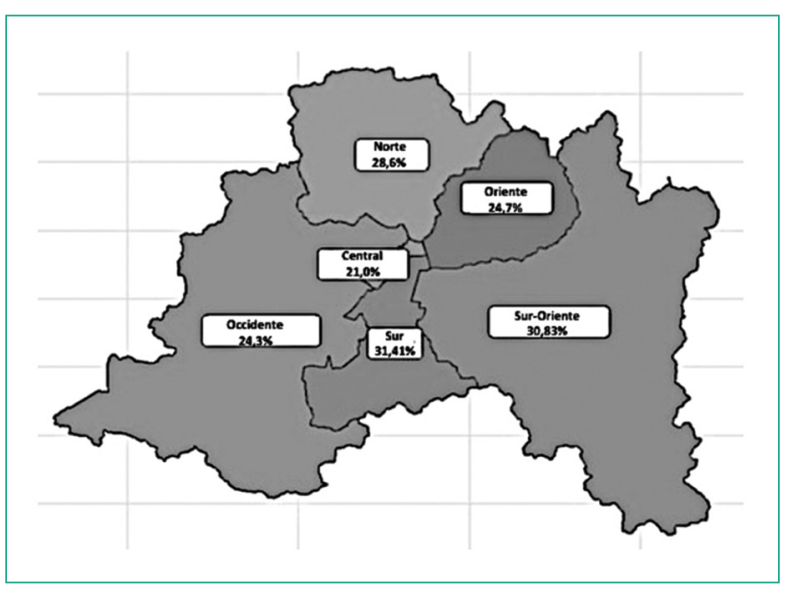

Figura 4. Distribución de letalidad de pacientes en hemodiálisis crónica, según el servicio de salud al que se pertenece.

mellitus, mientras que en trasplantados renales fueron edad sobre 60 años y bajo nivel socioeconómico (no mostrado).

Llamó la atención que la distribución geográfica de los casos influyó en el pronóstico de los pacientes con mayor letalidad en la región metropolitana (Santiago de Chile) donde fue del 27.7 vs. $17 \%$ en el resto de país. En la región metropolitana la letalidad de pacientes en HD con COVID-19 fue especialmente elevada (> 30\%) en la zona sur y sur-oriente (Fig. 4).

\section{Discusión}

El año 2020 ha presentado un desafío sin precedentes en las últimas décadas para la sociedad y los 
Tabla 3. Análisis multivariante de potenciales predictores de mortalidad en pacientes en hemodiálisis crónica en Chile durante primera ola de enfermedad por coronavirus 2019 (COVID -19) (marzo a diciembre de 2020). Se observa que la edad sobre 60 años, los meses en diálisis, tener diabetes mellitus y pertenecer a la región metropolitana son todos predictores de mayor riesgo de fallecer por COVID-19

\begin{tabular}{|l|c|c|c|c|}
\hline \multicolumn{5}{|c|}{ Análisis multivariante (regresión logística) de potenciales predictores de mortalidad } \\
\hline Variable & Odds ratio & $\begin{array}{c}\text { IC 95\% } \\
\text { límite inferior }\end{array}$ & $\begin{array}{c}\text { IC 95\% } \\
\text { límite superior }\end{array}$ & Valor de p \\
\hline Edad mayor de 60 años & 2.697 & 2.153 & 3.379 & $<0.001$ \\
\hline Tiempo en hemodiálisis & 1.002 & 1.001 & 1.004 & 0.001 \\
\hline Sexo masculino & 0.985 & 0.811 & 1.198 & 0.886 \\
\hline Diabetes & 1.580 & 1.287 & 1.940 & $<0.001$ \\
\hline Hipertensión & 0.976 & 0.763 & 1.247 & 0.847 \\
\hline Uso de inmunosupresores & 1.102 & 0.667 & 1.820 & 0.703 \\
\hline Centro privado & 0.753 & 0.504 & 1.125 & 0.166 \\
\hline Vivir en la región metropolitana & 1.677 & 1.353 & 2.083 & $<0.001$ \\
\hline
\end{tabular}

IC 95\%: intervalo de confianza del $95 \%$.

sistemas de salud en todo el mundo como resultado de la COVID-19, que fue declarada pandemia por la OMS en marzo de 2020. Pese a que se han invertido grandes esfuerzos en el desarrollo de estrategias y enfoques de mitigación para manejar la fase aguda de la enfermedad, así como en la investigación para proporcionar información sobre los mecanismos de la enfermedad y el desarrollo de vacunas actualmente en uso, las consecuencias de la pandemia de COVID-19 siguen siendo devastadoras ${ }^{19}$.

Es conocido que los pacientes con ERC tienen un alto riesgo de infección por SARS-CoV-2 y mortalidad asociada a COVID-19, en especial en los pacientes en $H D$, DP y Tx renal, quienes tienen un elevado riesgo de resultados adversos al desarrollar COVID-1920.

No existen dudas de que, en estos pacientes frágiles, una infección violenta que afecta los pulmones y el corazón, que ya están muy comprometidos en los pacientes en diálisis, los hace especialmente susceptibles a fallecer si desarrollan COVID-1920.

Por todo lo anterior en nuestro país desarrollamos la FUTAC Renal el 29 marzo del 2020, para evaluar todas las medidas basadas en la mejor evidencia posible, que pudieran mitigar el contagio y la mortalidad por esta enfermedad especialmente en los pacientes con ERC en TRR.

Este trabajo permitió además crear una base de datos nacional multicéntrica que reúne a más del $90 \%$ de los pacientes en HD, DP y Tx renales, pudiendo recolectar información valiosa de los resultados de estos pacientes durante la pandemia por COVID-19 durante el año 2020 (primera ola).

Como demuestran nuestros datos, los pacientes en HD son los de mayor riesgo de infección y mortalidad asociada a COVID-19. La tasa de infección en HD fue del $12.8 \%$ y la letalidad fue del $20 \%$ (7.3 veces mayor que la población general), lo que es concordante con publicaciones previas. Estos datos apoyan que los pacientes en HD constituyen una población de especial interés para desarrollar estrategias de prevención y vacunación. Los pacientes en DP y Tx renales pese a tener menor riesgo de contagio $(7.0 \%$ en DP y $5.2 \%$ en $\mathrm{Tx}$ renal), también presentan una elevada tasa de hospitalizaciones (en promedio $50 \%$ en cualquiera de las TRR), junto a una elevada letalidad (8.0 veces mayor en DP y 5.9 veces mayor en Tx renal que la población general), por lo que todos los pacientes con ERC en TRR deben considerarse en políticas públicas de prevención.

Como muestran nuestros datos al realizar un análisis multivariante de potenciales predictores de mortalidad en pacientes en HD crónica en Chile durante primera ola de COVID-19, la edad sobre 60 años, la antigüedad en $\mathrm{HD}$, la presencia de diabetes mellitus y el pertenecer a la región metropolitana son todos predictores de mayor riesgo de fallecer por COVID-19, razón por la que en este grupo particular de pacientes es donde se deben realizar las mayores estrategias de prevención y mitigación de la enfermedad. 
Es conocido que la pandemia de COVID-19 ha afectado especialmente a poblaciones de bajos ingresos socioeconómicos ${ }^{21}$, y en especial a las ciudades altamente pobladas, como lo es nuestra capital, Santiago de Chile, que fue efectivamente donde se observó la mayor tasa de infección y letalidad del país en los pacientes en HD. En un artículo realizado en población chilena se objetivó que la incidencia y mortalidad por COVID-19 en Santiago de Chile tiene un importante componente demográfico y socioeconómico ${ }^{22}$, razón por la cual quisimos evaluar este componente sindémico en la región metropolitana, realizando un análisis de la distribución de letalidad según los servicios de salud a donde pertenecían los pacientes. En la figura 4 se observa que la letalidad en la región metropolitana presentó una distribución geográfica heterogénea, evidenciándose una mayor letalidad en la zona sur y sur-oriente, donde superó el $30 \%$ de letalidad. Lo anterior puede explicarse por la mayor densidad demográfica y un menor estatus socioeconómico de estas zonas en Santiago de Chile ${ }^{21}$, apoyando un posible componente sindémico en los resultados clínicos de presentar COVID-19 en pacientes en HD.

Gracias a estos datos se pudo llegar a las autoridades de salud y lograr una vacunación prioritaria para este grupo de enfermos, encontrándose a la actualidad en Chile más del $90 \%$ de los pacientes con ERC en TRR con vacunación completa. El impacto de la vacunación en esta población con ERC en TRR está aún en evaluación por nuestro grupo.

Tenemos la convicción de que, en tiempos difíciles como el que hemos vivido, iniciativas como las descritas son del todo necesarias y debiesen ser consideradas un refuerzo a las medidas dictadas por las autoridades sanitarias del país.

Al sumar esfuerzos de organizaciones de pacientes, sociedades científicas y fundaciones hemos podido informar, capacitar, entregar recomendaciones y canalizar recursos económicos para ir en ayuda directa a pacientes y personal clínico, en un momento crucial y crítico para todos.

Es de suma importancia mantenernos unidos en el futuro pospandemia y seguir trabajando en equipo en beneficio de nuestros pacientes renales.

\section{Conclusiones}

Los pacientes con ERC en TRR que adquieren COVID-19 tienen mayor riesgo de contagio y mayor tasa de letalidad que la población general. En promedio la mitad de los pacientes en cualquiera de las TRR requieren hospitalización, siendo varias veces mayor la letalidad en este grupo que en la población general.

En los pacientes en HD los principales predictores de mortalidad fueron edad, diabetes, tiempo en la terapia y vivir en una zona altamente poblada como lo es la región metropolitana de Santiago de Chile. En los pacientes en DP fueron edad sobre 60 años, más de cinco años en la terapia y diabetes mellitus. En pacientes $T x$ renales fueron edad sobre 60 años y bajo nivel socioeconómico.

Destaca que en la región metropolitana que es la zona con mayor densidad poblacional del país, el sector geográfico donde residen los pacientes también puede influir en la letalidad por COVID-19, lo que puede apoyar que esta enfermedad tiene un componente sindémico en los pacientes en HD.

En suma, los pacientes con ERC en TRR deben considerarse de alto riesgo de contagiarse (en especial en HD) y de fallecer por esta enfermedad (todos los pacientes con ERC en TRR), por lo que se deben extremar todas las medidas basadas en evidencia para prevenir su contagio y considerarlos como una población prioritaria que debe recibir vacunación contra COVID-19.

Iniciativas como la FUTAC Renal son del todo necesarias para reforzar las medidas dictadas por las autoridades sanitarias del país en este grupo especial de pacientes.

\section{Agradecimientos}

Se agradece a todo el personal de enfermería que ayudó en el registro de estos datos: Hugo Arias Troncoso, Víctor Huenchulaf Vásquez, Elena Segovia Vega, Hugo Lazo Rejano, Pamela Cortez, Alejandra Chelme, Emperatriz Guevara Pizarro, Natalie Araya Cortés, Johanna Maita Hernández, Ana Payeros Rivera, Camila Serrano Barrera, Angélica Orrego Lazo, María José Fernández, Gloria Araya Aburto, Carolina Parra, GAV, Karina Rodríguez, Andrea Salgado Ávila, Mónica Arteaga, Ruth Bolados Rubio, Bella Velis Jaque, Verónica Campos Osses, Viviana Alfaro Castro y Karina Ponce Torres. Además a cada uno de los centros de diálisis públicos y privados que participaron en el registro de la FUTAC Renal de Chile: Complejo Asistencial Sótero del Río, Clínica Alemana Santiago, Diálisis Antares Talagante, Diálisis Calama, Centro médico y Diálisis Ltda. Sucursal Carmen Mena, Centro Médico y de Diálisis Ltda. Unidad Celia Zegers, CID Servicio Integral de Salud S.A., Centro de Diálisis Diamar, Hospital Militar, Centro de Diálisis de Mendoza Ltda., Diálisis Maiquén, Diálisis Municipal La Granja, Diálisis Ñuñoa, sucursal 
Quinta Normal, Diálisis Ñuñoa, Dialisis Ñuñoa Chacabuco, Diálisis Padre Hurtado de Cerrillos, Diálisis Antares Peñaflor, Diálisis Peñaflor, Diálisis San Juan de Dios, Centro de Diálisis San Lucas, Diálisis San Ramón, Centro de Diálisis Vespucio, Diálisis La Reina, Fresenius Chile, Diaverum Chile, Reddiálisis, Centro Renal SPA, Diálisis Lampa, Clínica Indisa, Clínica Santa María, Unidial S.A., Hospital Puerto Natales, Hospital Puerto Montt, Unidad Diálisis Modular Hospital Comunitario 21 de Mayo Taltal, Hospital Magallanes, Centro Diálisis Clínica Croacia Punta Arenas, Unidad de Diálisis Hospital Carlos Cisternas de Calama, Diálisis Nordial Ltda., Hospital Marcos Macuada Tocopilla, Diálisis Bupa Antofagasta, Centro Diálisis Ehrlich Ltda., Centro de Diálisis Hospital Comunitario de Mejillones, Diálisis Tecdial, Unidad de Diálisis Hospital Regional de Antofagasta, Centro de Diálisis Renacer, Centro Hemodiálisis crónica Hospital Clínico Universidad de Chile, Centro Hemodiálisis crónica UC-Christus, Hospital Regional Coyhaique, Hospital Puerto Aysen, Urodial, Nefrodial Molina, Diálisis del Libertador Rengo, Hospital regional Libertador Bernardo O'Higgins, Nefrodial Linares, Diálisis Aguamarina, Serdial Ltda., Centro de Diálisis Curicó Ltda., Nefrodial San Javier, Rancagua Dial Ltda., Diálisis San Isidro, Diálisis Gran Avenida, Premio Nobel, Vidadial Paillaco y Vidadial Lanco.

\section{Dedicatoria}

Esta publicación está dedicada al Dr. Andrés Boltansky Brenner, quien falleció en diciembre de 2020 a causa de la COVID-19. El Dr. Boltansky fue un nefrólogo muy dedicado a sus pacientes enfermos renales crónicos, en especial trasplantados renales, además de un activo miembro del directorio de la Sociedad Chilena de Nefrología y cofundador del equipo FUTAC.

\section{Financiamiento}

Los autores declaran no contar con financiamiento alguno.

\section{Conflicto de intereses}

Los autores declaran que no tienen conflicto de intereses.

\section{Responsabilidades éticas}

Protección de personas y animales. Los autores declaran que para esta investigación no se han realizado experimentos en seres humanos ni en animales.

Confidencialidad de los datos. Los autores declaran que han seguido los protocolos de su centro de trabajo sobre la publicación de datos de pacientes.

Derecho a la privacidad y consentimiento informado. Los autores declaran que en este artículo no aparecen datos de pacientes.

\section{Bibliografía}

1. Zhu N, Zhang D, Wang W, Li X, Yang B, Song J, et al. A novel coronavirus from patients with pneumonia in China, 2019. N Engl J Med. 2020;382(8):727-33.

2. Huang C, Wang Y, Li X, Ren L, Zhao J, Hu Y, et al. Clinical features of patients infected with 2019 novel coronavirus in Wuhan, China [published correction appears in Lancet. 2020 Jan 30]. Lancet. 2020;395(10223):497-506.

3. Hui DS, I Azhar E, Madani TA, Ntoumi F, Kock R, Dar O, et al. The continuing 2019-nCoV epidemic threat of novel coronaviruses to global health - The latest 2019 novel coronavirus outbreak in Wuhan, China. Int $\mathrm{J}$ Infect Dis. 2020;91:264-6.

4. Yi Y, Lagniton PNP, Ye S, Li E, Xu RH. COVID-19: what has been learned and to be learned about the novel coronavirus disease. Int J Biol Sci. 2020;16(10):1753-66.

5. Bernard Stoecklin S, Rolland P, Silue Y, First cases of coronavirus disease 2019 (COVID-19) in France: surveillance, investigations and control measures et al. First cases of coronavirus disease 2019 (COVID-19) in France: surveillance, investigations and control measures, January 2020. Euro Surveill. 2020;25(6):2000094.

6. Grasselli G, Zangrillo A, Zanella A, Antonelli M, Cabrini L, Castelli A, et al. Baseline characteristics and outcomes of 1591 patients infected with SARSCoV-2 admitted to ICUs of the Lombardy Region, Italy [published correction appears in JAMA. 2021;325(20):2120]. JAMA. 2020;323(16):1574-81.

7. Cucinotta D, Vanelli M. WHO declares COVID-19 a pandemic. Acta Biomed. 2020;91(1):157-60.

8. Ministerio de Salud de Chile. Reporte diarios de COVID-19 [Internet]. Chile: Ministerio de Salud. Disponible en: https://www.minsal.cl/wp-content/ uploads/2021/10/CP-REPORTE-COVID-19-Mi\%C3\%A9rcoles-131021.pdf

9. Hsu CM, Weiner DE. COVID-19 in dialysis patients: outlasting and outsmarting a pandemic. Kidney Int. 2020;98(6):1402-4.

10. Elias M, Pievani D, Randoux C, Louis K, Denis B, Delion A, et al. COVID-19 infection in kidney transplant recipients: Disease incidence and clinical outcomes. J Am Soc Nephrol. 2020;31(10):2413-23.

11. Ikizler TA. COVID-19 and dialysis units: What do we know now and what should we do? Am J Kidney Dis. 2020;76(1):1-3

12. Ma Y, Diao B, Lv X, Zhu J, Chen C, Liu L, et al. Epidemiological, clinical, and immunological features of a cluster of COVID-19-contracted hemodialysis patients. Kidney Int Rep. 2020;5(8):1333-41.

13. Ma Y, Diao B, Lv X, et al. COVID-19 in hemodialysis (HD) patients: Report from one HD center in Wuhan, China [Internet]. medR $x$ iv [24/02/2020]. Disponible en: https://www.medrxiv.org/content/10.1101/2020.02.24.20027201v3

14. Trujillo H, Caravaca-Fontán F, Sevillano A, Gutiérrez E, Caro J, Gutiérrez E, et al. SARS-CoV-2 infection in hospitalized patients with kidney disease. Kidney Int Rep. 2020;5(6):905-9.

15. Alberici F, Delbarba E, Manenti C, Econimo L, Valerio F, Pola A, et al. Management of patients on dialysis and with kidney transplantation during the SARS-CoV-2 (COVID-19) pandemic in Brescia, Italy. Kidney Int Rep. 2020;5(5):580-5.

16. Sosa R, Garcia P, Cipriano EO, Hernández A, Hernández EE, Chavez PI, et al. Coronavirus disease 2019 in patients with end-stage kidney disease on hemodialysis in Guatemala. Kidney Int Rep. 2021;6(4):1110-7.

17. Fuerza de Trabajo Anti Covid-19-FUTAC Renal. COVID al día. Últimas novedades [Internet]. Sociedad Chilena de Nefrología [acceso: 16/06/2021]. Disponible en: https://www.nefro.cl/covid

18. Torres Díaz R, Lorca Herrera E. Covid-19 en pacientes renales crónicos: llamado de atención. Rev Med Chil. 2020;148(5):711-2.

19. Bruchfeld A. The COVID-19 pandemic: consequences for nephrology. Nat Rev Nephrol. 2021;17(2):81-2.

20. Rombolà G, Brunini F. COVID-19 and dialysis: why we should be worried. J Nephrol. 2020;33(3):401-3.

21. Bong CL, Brasher C, Chikumba E, McDougall R, Mellin-Olsen J, Enright A. The COVID-19 pandemic: Effects on low- and middle-income countries. Anesth Analg. 2020;131(1):86-92.

22. Mena GE, Martinez PP, Mahmud AS, Marquet PA, Buckee CO, Santillana M. Socioeconomic status determines COVID-19 incidence and related mortality in Santiago, Chile. Science. 2021;372(6545):eabg5298. 